VIEWPOINT

\title{
Can the published cost analysis data for delivery of an efficient primary angioplasty service be applied to the modern National Health Service?
}

\author{
N Melikian, K P Morgan, K J Beatt
}

Heart 2005;91:1262-1264. doi: 10.1136/hrt.2004.059402

Despite the clinical benefits and safety profile of primary percutaneous coronary intervention (PCI), the health care system in the UK has been slow to adopt this strategy as first line management for ST segment elevation myocardial infarction. The cost implications of a 24 hour a day, seven days per week primary $\mathrm{PCl}$ service and the absence of an existing efficient working model within the National Health Service (NHS) framework are two of the major deterrents for provision of such a service. The existent cost effectiveness data for primary $\mathrm{PCl}$ is critically reviewed, with particular reference to the NHS.

See end of article for authors' affiliations

Correspondence to: Dr K Beatt, Cardiac Catheter Suite, Hammersmith Hospital, Du Cane Road, London W12 OHS, UK; k.beatt@ imperial.ac.uk compared with thrombolysis. However critics of primary PCI in the UK remain sceptical and continue to question the applicability of these models to the NHS. The National Service Framework for coronary artery disease has acknowledged the unresolved "implications of introducing and maintaining a 24/7 primary mended the setting up of primary PCI models to explore the unanswered financial questions.

\section{THE EXISTING EVIDENCE \\ Europe}

A number of studies from the Netherlands have addressed the cost effectiveness of primary PCI. ${ }^{2-4}$ The first was the Zwolle trial where STEMI patients were randomised to receive either streptokinase or primary PCI and followed up for a minimum of 12 months. ${ }^{2}$ Although the final calculations demonstrated that thrombolysis was cheaper in the acute setting, this difference had disappeared over a 12 month period (cost per patient at 12 months: thrombolysis \$16 681 and primary PCI \$17 316; $\mathrm{p}=0.22$ ). Furthermore, the cost effectiveness ratio (average cost per event-free survivor) of primary PCI at \$25 431 per patient was significantly less than the \$36798 accrued for each thrombolysis patient. Similarly, indirect costs such as the bill for pharmacological agents were also significantly lower for primary PCI (primary PCI $\$ 695$ and streptokinase $\$ 854$ per patient; $\mathrm{p}=0.01)^{2}$

Other groups from the Netherlands have addressed the cost of stent usage in the context of primary PCI, by randomising STEMI patients to balloon angioplasty alone (POBA) or balloon angioplasty followed by stent deployment (stent). ${ }^{3}$ The results mimicked those obtained in the Zwolle trial. ${ }^{3}$ For example, in the study by Suryapranata and colleagues, stent deployment incurred higher initial costs, but by 24 months the cumulative costs between the two groups were comparable. ${ }^{3}$ The mean cost effectiveness ratio (as calculated in one of the two studies) also favoured stenting (Dfl 37 408) over POBA (Dfl 53 117; $\mathrm{p}<0.001$ ). The investigators attributed the comparable cost of the two treatment modalities to the better clinical outcome of patients who had received stents, resulting in lower repeat revascularisation rates (target vessel revascularisation: $12 \%$ stent $v \quad 34 \%$ POBA; $\mathrm{p}<0.001$ ) and recurrent cardiac events (cardiac event free survival: $84 \%$ stent $v 62 \%$ POBA; $\mathrm{p}<0.001)^{3}$ angioplasty" service and in turn has recom-
Abbreviations: NHS, National Health Service; $\mathrm{PCl}$, percutaneous coronary intervention; PAMI, primary angioplasty in myocardial infarction; $\mathrm{POBA}$, plain old balloon angioplasty; rt-PA, recombinant tissue plasminogen activator; STEMI, ST elevation myocardial infarction; QALY, quality adjusted life-year 


\section{North America}

Despite major differences in the delivery of heath care between Europe and North America, the cost effectiveness data from primary PCI studies appear to be broadly similar. ${ }^{5-7}$ A major difference between North American and European studies is the substitution of hospital charges for actual costs incurred by the health service. Breakdown of hospital charges indicates that professional fees (which is not a feature of most national health care systems in Europe) form a significant component of the overall charge and can in some cases exceed the actual costs borne by the health service.

The Mayo Clinic trial randomly compared thrombolysis with recombinant tissue plasminogen activator (rt-PA) with primary PCI. ${ }^{5}$ In the short term there was a non-significant trend towards lower costs in the primary PCI patients (total charge per patient: rt-PA $\$ 28$ 600, PCI $\$ 24$ 900; $p=0.15$ ). However, when all indirect measures of cost, including late procedures and readmissions were accounted for, primary PCI was clearly more cost effective. Breakdown of costs in the PAMI (primary angioplasty in myocardial infarction) trial also replicated previous findings, where per patient hospital charges (excluding professional fees) were significantly lower in primary PCI patients. ${ }^{6}$ The PAMI investigators demonstrated that the greatest cost reduction with primary PCI was achieved in the low risk (as defined by TIMI (thrombolysis in myocardial infarction) criteria) subgroup of patients (a mean saving of $\$ 4365$ per patient), who were discharged on average 1.3 days earlier than the thrombolysis group. In the high risk subgroup of patients there was only a non-significant trend towards a lower overall cost with primary PCI. The strongest predictor of cost in this study was the duration of hospital stay. The more recently published Canadian STAT study, which randomised acute STEMI patients to primary stenting (stent) or rt-PA, also demonstrated significantly lower costs for the initial hospitalisation (stent $\$ 6354 \pm \$ 6382$, rt-PA $\$ 7893 \pm \$ 4429 ; p=0.001$ ), as well as at six months (stent $\$ 7100 \pm \$ 711$, rt-PA $\$ 9559$ \pm \$6933; $\mathrm{p}=0.001$ ) in the invasive arm of the study. ${ }^{7}$

Table 1 summarises the five major publications discussed.

\section{Models of primary $\mathrm{PCl}$}

Health economists have also produced complex "decision analytic models" to complement the cost effectiveness data generated from actual primary PCI trials. Unlike randomised trials with highly selective inclusion criteria, these models have utilised health outcome information from a broad selection of sources to create balanced hypothetical scenarios in order to provide a comparison of the costs between primary PCI and thrombolysis.

In the model proposed by Lieu and colleagues for 10000 STEMI patients, projections demonstrated a significant reduction in overall cost and quality adjusted life-years (QALYs) saved in favour of primary PCI. ${ }^{8}$ The cost per life saved was \$11000 for primary PCI and \$14000 for thrombolysis. The model also accounted for the increased cost of using stents for primary PCI, demonstrating that over a 12 month period an estimated $20 \%$ increase in overall cost per patient would be balanced by significant reduction in continued costs-for example, repeat procedures, admissions, and the cost for pharmacological agents. ${ }^{8}$

The authors of this model outline important prerequisites for the running of an efficient cost effective primary PCI programme, including: (1) use of an existent cardiac catheterisation laboratory; (2) a throughput of at least 200 cases per annum; (3) a long term view of providing a primary PCI service (at least six years); and (4) utilisation of existent on call technical/nursing staff and cardiothoracic support teams. In the proposed model the cost effectiveness of the primary PCI programme was highly dependent on the volume of work and the length of time for which the service

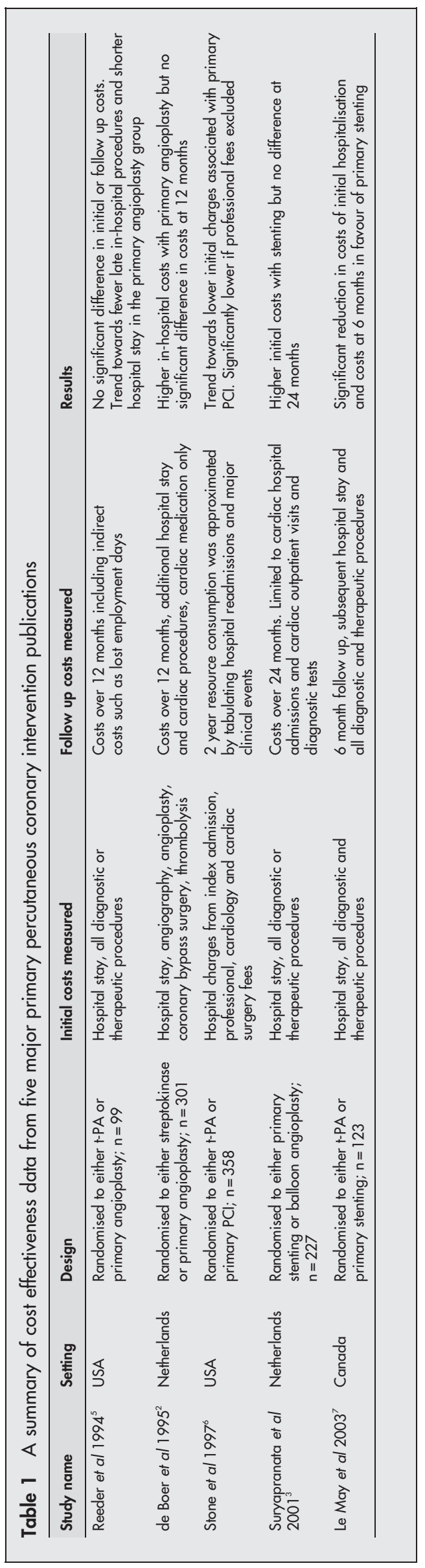


was provided. Costs increased exponentially if the number of patients treated fell below 100 cases per annum and if the service was run for less than six years. ${ }^{8} 9$

\section{THE NHS IN 2005}

The fundamental question is whether primary PCI data from other healthcare systems and health economical models can be applied to the NHS? To answer this very question the Department of Health has pledged in excess of $£ 1$ million to set up a number of investigative primary PCI programmes around the country. While actual cost effectiveness data are awaited we have critically analysed the applicability of the existent information to the NHS.

Although the broad concepts from existent primary PCI studies remain applicable to the NHS, the information on outcome and cost effectiveness are outdated and are in need of further scrutiny. Since the publication of the majority of the primary PCI cost effectiveness data in the mid 1990s, interventional cardiology has undergone significant changes. Novel techniques, equipment, designs, and adjuvant treatments (such as the antiplatelet agents clopidogrel and abciximab) over the past decade have all contributed to major improvements in both acute and long term outcomes with reductions in repeat revascularisation, readmission, and mortality rates. Furthermore, in this period the UK has witnessed a major increase in the number of coronary interventional procedures leading to a substantial reduction in the cost of PCI. Therefore, re-evaluation of any one of the models we have described using current price tags and projected outcomes should in theory increase effectiveness of primary PCI in comparison to thrombolysis. It is also vital to note that thrombolysis has undergone minimal change during the same time frame. Second and third generation tissue plasminogen derivatives, which are now increasingly used by health care trusts in the UK, remain expensive and their mid to long term clinical outcome inferior to that of primary PCI.

It is also important to note that all cost analyses data to date are derived from randomised controlled trials with stringent inclusion and exclusion criteria. Therefore, information from a real world primary PCI service is vital in order to have a meaningful analysis of the cost of running a comprehensive primary PCI service.

Adherence to the four criteria as proposed by Lieu and colleagues on volume of workload and the use of existent cardiac facilities are vital and highly applicable to the NHS. ${ }^{89}$ Primary PCI programmes within the NHS must make use of existing facilities and there is a need to explore more efficient systems by optimising the volume and concentration of expertise in designated regional STEMI centres. Furthermore, the use of these facilities, which already have complex staffing arrangements to provide round the clock catheterisation and cardiothoracic services, should prevent the spiralling of staffing costs. However, the applicability of such models to the rural parts of the country remains questionable, where alternative reperfusion strategies such as prehospital thrombolysis and/or a hybrid thrombolysis-PCI programme may be of greater value.

\section{Do we have a possible future model for an efficient NHS primary $\mathrm{PCl}$ programme?}

The North-West London Primary PCI Programme has been set up to investigate some of the outstanding issues regarding the cost effectiveness of primary PCI within the framework of the NHS. The programme utilises the principles outlined in previously published health economic models. ${ }^{8}{ }^{9}$ It is based on a dynamic "hub and spoke" model, with one established central tertiary cardiac centre and three high volume district general hospitals. The London Ambulance Service is used for rapid direct and/or inter-hospital transfer of acute STEMI patients. Since its inception in October 2003 the service has been rolled out in a stepwise manner to surrounding hospitals and represents the first comprehensive regional 24/7 primary PCI service. Currently it treats on average a minimum of one patient every day.

Meticulous cost analysis, including information on the cost savings achieved with reductions in length of hospital stay, re-admission and repeat procedure rates, as well as information on medium to long term complications should over the next two years provide accurate information on the viability of this model as a 24/7 urban NHS primary PCI programme. Furthermore, these data will be the first "real world, nontrial" evidence of outcomes and costs for a comprehensive primary PCI programme.

\section{Authors' affiliations}

N Melikian*, K Morgan, K J Beatt, Cardiology Department,

Hammersmith Hospital, London, UK

*Also Department of Cardiovascular Medicine, GKT School of Medicine, King's College Hospital, London, UK

\section{REFERENCES}

1 The National Service Framework for coronary heart disease - winning the war on heart disease. Progress report 2004.

2 De Boer MJ, van Hout BA, Liem AL, et al. A cost-effective analysis of primary coronary angioplasty versus thrombolysis for acute myocardial infarction. Am J Card 1995;76:830-3.

3 Suryapranata H, Ottervanger JP, Nibbering E, et al. Long term outcome and cost-effectiveness of stenting versus balloon angioplasty for acute myocardial infarction. Heart 2001;85:667-71.

4 Van't Hof AW, Suryapranata H, de Boer MJ, et al. Costs of stenting for acute myocardial infarction. Lancet 1998;351:1817.

5 Reeder GS, Bailey KR, Gersh BJ, et al. Cost comparison of immediate angioplasty versus thrombolysis followed by conservative therapy for acute myocardial infarction: a randomised prospective trial. Mayo Clin Proc 1994;69:5-12

6 Stone GW, Grines CL, Rothbaum D, et al. Analysis of the relative costs and effectiveness of primary angioplasty versus tissue-type plasminogen activator: the primary angioplasty in myocardial infarction (PAMI) trial. J Am Coll Cardiol 1997;29:901-7.

7 Le May MR, Davies RF, Labinaz M, et al. Hopsitalization costs of primary stenting versus thrombolysis in acute myocardial infarction - cost analysis of the Canadian STAT study. Circulation 2003; 108:2624-30.

8 Lieu TA, Gurley RJ, Lundstrom RJ, et al. Projected cost-effectiveness of primary angioplasty for acute myocardial infarction. J Am Coll Cardiol 1997:30:1741-50.

9 Parmley WW. Cost-effectiveness of reperfusion strategies. Am Heart J $1999 ; 138:$ S1 42-6. 